Vol. 43 (1991) [115-122]

\title{
LEFT IDEALS IN THE NEAR-RING OF AFFINE TRANSFORMATIONS
}

\section{WOLFGaNg MUTter}

\begin{abstract}
In this paper we determine the left ideals in the near-ring $A f f(V)$ of all affine transformations of a vector space $V$. It is shown that there is a Galois correspondence between the filters of affine subspaces of $V$ and those left ideals of $\operatorname{Aff}(V)$ which are not left invariant. In particular, the not left invariant finitely generated left ideals of $\operatorname{Aff}(V)$ are precisely the annihilators of the affine subspaces of $V$. A similar correspondence exists between the filters of linear subspaces of $V$ and the left invariant left ideals of $A f(V)$. If $V$ is finite-dimensional, then all left ideals of $\operatorname{Aff}(V)$ are finitely generated.
\end{abstract}

\section{INTRODUCTION}

Let $V$ be a vector space and let $\operatorname{Aff}(V)$ denote the collection of all affine transformations of $V$. Under pointwise addition and under composition of mappings $A f f(V)$ is a near-ring. In [2] Blackett showed that the set $C$ of all constant transformations forms an ideal of $\operatorname{Aff}(V)$. If $V$ is finite dimensional, then $C$ is the only non-trivial ideal of $\operatorname{Aff}(V)$. Wolfson [5] determined all ideals of $\mathrm{Aff}(V)$ for an arbitrary vector space $V$. He observed that $C$ is contained in all non-trivial ideals of $\mathrm{Aff}(V)$ and that $\mathrm{Aff}(V) / C$ is isomorphic to the ring $\operatorname{Hom}(V, V)$ of all linear transformations of $V$. Thus the ideals of Aff $(V)$ are the sets $T_{\nu}+C$ with $T_{\nu}=\left\{f \in \operatorname{Hom}(V, V) \mid \operatorname{Range} f<\aleph_{\nu}\right\}$, where $\aleph_{\nu}$ is a cardinal number.

In this paper we investigate the structure of the left ideals of $\mathrm{Aff}(V)$. We use the results of Baer on the left ideals of the ring $\operatorname{Hom}(V, V)$ in $[1$, p.172 following], where he showed that the finitely generated left ideals of $\operatorname{Hom}(V, V)$ are precisely the annibilators of the linear subspaces of the vector space $V$. In particular, Baer established a Galois correspondence between the left ideals of $\operatorname{Hom}(V, V)$ and the filters of linear subspaces of $V$. Thus, by the second isomorphism theorem for near-rings (see for example Theorem 1.31 in [3]), the left invariant left ideals of $A f f(V)$ are completely determined, since a left ideal of $\operatorname{Aff}(V)$ is left invariant if and only if it contains the ideal $C$ of all constant transformations of $V$.

The purpose of this paper is to show that there is a similar correspondence between the left ideals of $\operatorname{Aff}(V)$ which are not left invariant and the affine subspaces of $V$, as

Received 21 February 1990

Copyright Clearance Centre, Inc. Serial-fee code: 0004-9729/91 \$A2.00+0.00. 
in the case of $\operatorname{Hom}(V, V)$. If $V$ is finite dimensional, then all left ideals of $\operatorname{Aff}(V)$ are finitely generated. In this case the left ideals of $A f f(V)$ which are not left invariant are precisely the annihilators of the affine subspaces of $V$. The left invariant left ideals of $\operatorname{Aff}(V)$ are the sets $L+C$, where $L$ is the annihilator of a linear subspace of $V$.

\section{BASIC DEFINITIONS AND RESULTS}

For details on near-rings and $N$-groups we refer the reader to [4]. According to [4] we consider right near-rings.

Definition 2.1: Let $(N,+, \cdot)$ be a near-ring. A subset $L$ of $N$ is called a left ideal of $N$ provided that

1. $(L,+)$ is a normal subgroup of $(N,+)$, and

2. $m(n+i)-m n \in L$ for all $i \in L$ and $m, n \in N$.

If $S$ is a subset of a near-ring $N$, let $\langle S\rangle_{\ell}$ denote the left ideal generated by $S$. In particular, $\left\langle n_{1}, \ldots, n_{k}\right\rangle_{\ell}$ denotes the left ideal generated by $n_{1}, \ldots, n_{k} \in N$. If a near-ring $N$ is regarded as a $N$-group in the usual way, the left ideals of $N$ are precisely the kernels of $N$-homomorphisms with domain $N$.

In general, a left ideal of a near-ring is not invariant under multiplication from the left. Therefore, we call a left ideal $L$ of a near-ring $N$ left invariant, if for all $n \in N$ and $i \in L$ the element $n \cdot i$ is in $L$. The left invariant left ideals of a near-ring can be characterised as follows:

Lemma 2.2. Let $N$ be a near-ring with constant part $N_{c}$ and let $L$ be a left ideal of $N$. Then $L$ is left invariant if and only if $N_{c} \subseteq L$.

PROOF: If $L$ is left invariant and $n_{c}$ is in $N_{c}$, then $n_{c}=n_{c} \cdot i \in L$ for all $i \in L$. Conversely, if $N_{c} \subseteq L$, then for all $n \in N$ and $i \in L$ the element $n \cdot i=n \cdot i-n \cdot 0+n \cdot 0$ is in $L$, since $n \cdot 0$ is in $N_{c}$.

If $V$ is a vector space and $S$ is a subset of $V$, then $\operatorname{Ann}(S)$ denotes the annihilator $\{f \in \operatorname{Aff}(V) \mid f(S)=0\}$. If $p$ is an element of $V$, let $\langle p\rangle$ denote the constant transformation of $V$ which carries all of $V$ onto $p$. Any affine transformation $f \in$ $\operatorname{Aff}(V)$ can be decomposed as $f=f-\langle f(0)\rangle+\langle f(0)\rangle$ with $f-\langle f(0)\rangle \in \operatorname{Hom}(V, V)$ and $(f(0)) \in C$. Hom $(V, V)$ is a subnear-ring of $\operatorname{Aff}(V)$ and

$$
\varphi: \operatorname{Aff}(V) \rightarrow \operatorname{Hom}(V, V): f \mapsto f-\langle f(0)\rangle
$$

is a surjective near-ring homomorphism with $\operatorname{ker} \varphi=C$. By Lemma 2.2 and by the second isomorphism theorem for near-rings ([3, Theorem 1.31]) $\varphi$ induces a bijective correspondence between the left invariant left ideals of $\mathrm{Aff}(V)$ and the left ideals of $\operatorname{Hom}(V, V)$ by $L \rightarrow \varphi(L)$. 
A left ideal $L$ of $\mathrm{Aff}(V)$ which is not left invariant does not contain many constant transformations, for we have

Lemma 2.3. If $L$ is a not left invariant left ideal of $\mathrm{Aff}(V)$, then $L \cap C=\{0\}$.

Proof: It is easy to show that $L \cap C$ is isomorphic to a submodule of the simple $\operatorname{Hom}(V, V)$-module $V$. Hence, by Lemma 2.2, the assertion of the lemma is obvious.

For an affine transformation $f$ let $Z(f)$ denote the zero-set of $f$, that is $Z(f)=$ $\{p \in V \mid f(p)=0\}$. If $Z(f)$ is not empty, then it is an affine subspace of $V$. Conversely, every affine subspace of a vector space is the zero-set of an affine transformation. More precisely:

Lemma 2.4. Let $A=p+U$ be an affine subspace of a vector space $V$, where $U$ is a linear subspace of $V$ and $p \in V$. Then there exists $f \in \operatorname{Aff}(V)$ with $Z(f)=A$. In particular, if $W$ is a linear complement of $U$ in $V$, there exists $f \in \operatorname{Aff}(V)$ with $Z(f)=A$ and $f(V)=W$.

Proof: By the Complementation Theorem in [1, p.12], there exists a linear subspace $W$ of $V$ with $V=U \oplus W$. If $\tau_{-p}$ denotes the translation by $-p$ and $p r_{W}$ is the projection map from $V$ onto $W$, then $f=p r_{W} \circ \tau_{-p}$ is an affine transformation of $V$ with the required properties.

\section{THE NOT LEFT INVARIANT LEFT IDEALS}

In this section we determine the left ideals of $\mathrm{Aff}(V)$ which are not left invariant.

Lemma 3.1. Let $L$ be a left ideal of $\operatorname{Aff}(V)$ and let $f_{1}, \ldots, f_{n}$ be in $L$ with $Z\left(f_{1}\right) \cap \cdots \cap Z\left(f_{n}\right) \neq \emptyset$. If $g$ is an affine transformation of $V$ with $Z(g) \supseteq Z\left(f_{1}\right) \cap$ $\cdots \cap Z\left(f_{n}\right)$, then $g \in L$.

Proof: Since $Z\left(f_{1}\right) \cap \cdots \cap Z\left(f_{n}\right)$ is not empty, there exist an element $p \in V$ and a linear subspace $U$ of $V$ with $p+U=Z\left(f_{1}\right) \cap \cdots \cap Z\left(f_{n}\right)$. Let $\tau_{p} \in \mathrm{Aff}(V)$ be given by $\tau_{p}(x)=x+p$. Then $\tau_{p}$ defines an Aff(V)-automorphism of Aff(V) by $h \mapsto h \circ \tau_{p}$. Hence

$$
U=Z\left(f_{1} \circ \tau_{p}\right) \cap \cdots \cap Z\left(f_{n} \circ \tau_{p}\right)
$$

and $U \subseteq Z\left(g \circ \tau_{p}\right)$. In particular, $f_{1} \circ \tau_{p}, \ldots, f_{n} \circ \tau_{p}$ and $g \circ \tau_{p}$ are linear transformations of $V$. Since $\operatorname{Hom}(V, V)$ is a left ideal of $\operatorname{Aff}(V)$, the left ideal $\left(f_{1} \circ \tau_{p}, \ldots, f_{n} \circ \tau_{p}\right\rangle_{l}$ generated by $f_{1} \circ \tau_{p}, \ldots, f_{n} \circ \tau_{p}$ is obviously the smallest left ideal of the ring $\operatorname{Hom}(V, V)$ which contains $f_{1} \circ \tau_{p}, \ldots, f_{n} \circ \tau_{p}$. Hence $g \circ \tau_{p} \in\left\langle f_{1} \circ \tau_{p}, \ldots, f_{n} \circ \tau_{p}\right\rangle_{\ell}$ by $[1$, p.173, Theorem A, and p.177, Theorem 1]. The second isomorphism theorem 1.30 for $N$. groups in [4] implies $g \in\left(f_{1}, \ldots, f_{n}\right\rangle_{\ell} \subseteq L$.

In order to prove the next lemma, we need the following two propositions: 
Proposition 3.2. Let $V$ be a vector space and let $A_{1}$ and $A_{2}$ be affine subspaces of $V$ with $A_{1} \cap A_{2}=\emptyset$. Then there exist maximal affine subspaces $M_{1}$ and $M_{2}$ of $V$ such that $A_{1} \subseteq M_{1}, A_{2} \subseteq M_{2}$ and $M_{1} \cap M_{2}=\emptyset$.

Proof: Let $p_{1}, p_{2}$ be in $V$ and let $U_{1}, U_{2}$ be linear subspaces of $V$ with $A_{1}=$ $p_{1}+U_{1}$ and $A_{2}=p_{2}+U_{2}$. Since $A_{1} \cap A_{2}=\emptyset, p_{1}-p_{2}$ is not in $U_{1}+U_{2}$. By the Complementation Theorem in [1, p.12], there exists a linear subspace $U$ of $V$ such that $V$ can be decomposed as

$$
V=\operatorname{span}\left(p_{1}-p_{2}\right) \oplus\left(U_{1}+U_{2}\right) \oplus U .
$$

Then $M_{1}=p_{1}+\left(U_{1}+U_{2}+U\right)$ and $M_{2}=p_{2}+\left(U_{1}+U_{2}+U\right)$ are maximal affine subspaces of $V$ with the required properties.

Proposition 3.3. If $L$ is a left ideal of $\mathrm{Aff}(V)$ and $f \in L$ with $Z(f)=\emptyset$, then $L$ is left invariant.

Proof: $f(V)$ is an affine subspace of $V$. Thus by Lemma 2.4 there exists $g \in$ $\operatorname{Aff}(V)$ with $Z(g)=f(V)$. Furthermore the constant transformation

$$
\langle-g(0)\rangle=g \circ f-g \circ\langle 0\rangle
$$

is in $L$. Moreover, $g(0)$ is not zero, since 0 is not in $f(V)$. Hence the assertion of the lemma follows by Lemmas 2.2 and 2.3 .

Lemma 3.4. Let $L$ be a left ideal of $\operatorname{Aff}(V)$ and suppose there are $f, g \in L$ with $Z(f) \cap Z(g)=\emptyset$. Then $L$ is left invariant.

Proof: By Proposition 3.3 it suffices to show that there exists an affine transformation $h \in L$ with $Z(h)=\emptyset$. Therefore we may assume that $Z(f)$ and $Z(g)$ are not empty. By Proposition 3.2 there exist maximal subspaces $M_{1}$ and $M_{2}$ of $V$ such that $Z(f) \subseteq M_{1}, Z(g) \subseteq M_{2}$ and $M_{1} \cap M_{2}=\emptyset$. By Lemma 2.4 there exist nonzero elements $p_{1}$ and $p_{2}$ in $V$ and transformations $f_{1}, f_{2} \in \operatorname{Aff}(V)$ with $M_{1}=Z\left(f_{1}\right), M_{2}=Z\left(f_{2}\right)$, $f_{1}(V)=\operatorname{span}\left(p_{1}\right)$ and $f_{2}(V)=\operatorname{span}\left(p_{2}\right)$. Lemma 3.1 implies $f_{1}, f_{2} \in L$, since $Z\left(f_{1}\right) \supseteq Z(f)$ and $Z\left(f_{2}\right) \supseteq Z(g)$. Now we distinguish two cases:

Suppose $\operatorname{dim} V>1$. Then there exist nonzero elements $q_{1}, q_{2} \in V$ with $\operatorname{span}\left(q_{1}\right) \cap \operatorname{span}\left(q_{2}\right)=\{0\} . \quad$ Let $h_{1}$ and $h_{2}$ be invertible linear transformations of $V$ with $h_{1}\left(p_{1}\right)=q_{1}$ and $h_{2}\left(p_{2}\right)=q_{2}$. Then $h_{1} \circ f_{1}(V)=\operatorname{span}\left(q_{1}\right)$ and $h_{2} \circ f_{2}(V)=\operatorname{span}\left(q_{2}\right)$. Furthermore the transformation $h=h_{1} \circ f_{1}-h_{2} \circ f_{2}$ is in $L$. If $x \in V$, then

$h(x)=0 \Leftrightarrow h_{1} \circ f_{1}(x)=h_{2} \circ f_{2}(x) \Leftrightarrow h_{1} \circ f_{1}(x)=h_{2} \circ f_{2}(x)=0 \Leftrightarrow f_{1}(x)=f_{2}(x)=0$.

Hence $Z(h)=\emptyset$, since $Z\left(f_{1}\right) \cap Z\left(f_{2}\right)=\emptyset$. This proves the assertion of the lemma for $\operatorname{dim} V>1$. 
If $\operatorname{dim} V=1$, then there exist distinct elements $q_{1}$ and $q_{2}$ in $V$ with $Z\left(f_{1}\right)=\left\{q_{1}\right\}$ and $Z\left(f_{2}\right)=\left\{q_{2}\right\}$. An easy check shows that in this case $f_{1}$ and $f_{2}$ are injective. Hence there exist affine transformations $h_{1}$ and $h_{2}$ with $h_{1} \circ f_{1}=h_{2} \circ f_{2}=i d$. The constant transformation

$$
h=\left\langle g_{2}(0)-g_{1}(0)\right\rangle=\left(g_{1} \circ f_{1}-g_{1} \circ\langle 0\rangle\right)-\left(g_{2} \circ f_{2}-g_{2} \circ\langle 0\rangle\right)
$$

is in $L$ and is not zero, since $h_{1}(0)=h_{1}\left(f_{1}\left(q_{1}\right)\right)=q_{1}$ and $h_{2}(0)=h_{2}\left(f_{2}\left(q_{2}\right)\right)=q_{2}$. This completes the proof of the lemma.

Now we are in a position to establish a bijective correspondence between the left ideals of $\operatorname{Aff}(V)$, which are not left invariant, and the filters of affine subspaces of $V$. First we need

DEFINITION 3.5: A nonempty family $\mathcal{F}$ of affine subspaces of a vector space $V$ is called an $\mathcal{A}$-filter on $V$ provided that

1. $\emptyset \notin \mathcal{F}$,

2. if $A_{1}, A_{2} \in \mathcal{F}$, then $A_{1} \cap A_{2} \in \mathcal{F}$, and

3. if $A \in \mathcal{F}$ and $A^{\prime}$ is an affine subspace of $V$ with $A^{\prime} \supseteq A$, then $A^{\prime} \in \mathcal{F}$.

For example, if $A$ is an affine subspace of $V$, the family $\mathcal{F}_{A}$ of all affine subspaces of $V$ which contain $A$ is an $\mathcal{A}$-filter on $V$. Obviously $\mathcal{F}_{A}$ is the smallest $\mathcal{A}$-filter containing $A$, hence we call $\mathcal{F}_{A}$ the $\mathcal{A}$-filter generated by $A$.

THEOREM 3.6. Let $V$ be a vector space.

1. If $L$ is a left ideal of $\mathrm{Aff}(V)$ which is not left invariant, then

$$
Z[L]=\{Z(f) \mid f \in L\}
$$

is an $\mathcal{A}$-filter on $V$.

2. If $\mathcal{F}$ is an $\mathcal{A}$-filter on $V$, then

$$
Z \leftarrow[\mathcal{F}]=\bigcup\{\operatorname{Ann}(A) \mid A \in \mathcal{F}\}
$$

is a not left invariant left ideal of $\mathrm{Aff}(V)$.

Moreover, the mapping $Z$ is one-one between the set of all not left invariant left ideals of $\operatorname{Aff}(V)$ and the $\mathcal{A}$-filters on $V$.

Proof: 1 . Let $L$ be a left ideal of $\operatorname{Aff}(V)$ which is not left invariant. We have to show that $Z[L]$ satisfies the properties $1-3$ of Definition 3.5. Proposition 3.3 implies $\emptyset \notin Z[L]$. Suppose now that $A_{1}, A_{2} \in Z[L]$. If $A_{1} \cap A_{2}=\emptyset$, then by Lemma $3.4 L$ is left invariant, which contradicts the hypothesis. If $A_{1} \cap A_{2} \neq \emptyset$, then according to Lemma 2.4 there exists $f \in \operatorname{Aff}(V)$ with $Z(f)=A_{1} \cap A_{2}$. Lemma 3.1 implies $f \in L$, 
hence $A_{1} \cap A_{2} \in Z[L]$. Finally, let $A \in Z[L]$ and let $A^{\prime}$ be an affine subspace of $V$ with $A^{\prime} \supseteq A$. By Lemma 2.4 there exists $f^{\prime} \in \mathrm{Aff}(V)$ with $A^{\prime}=Z\left(f^{\prime}\right)$. Since $A \neq \emptyset$ by Lemma 3.4, Lemma 3.1 implies $f^{\prime} \in L$. Therefore $A^{\prime}$ is in $Z[L]$. Altogether, we have shown that $Z[L]$ is an $\mathcal{A}$-filter on $V$.

2. The proof of the second assertion of the theorem is straightforward and therefore omitted.

3. In order to verify that the mapping $Z$ is one-one, we prove that $Z^{-}$is the inverse mapping of $Z$. If $\mathcal{F}$ is an $\mathcal{A}$-filter on $V$ then clearly $Z\left[Z^{\leftarrow}[\mathcal{F}]\right]=\mathcal{F}$. Furthermore it is obvious that any left ideal $L$ of $\operatorname{Aff}(V)$ satisfies $L \subseteq Z^{-}[Z[L]]$. If, in addition, $L$ is not left invariant, we have seen that $Z[L]$ is an $\mathcal{A}$-filter on $V$. Therefore, if $f$ is an affine transformation with $Z(f) \in Z[L]$, then $Z(f) \neq \emptyset$, and hence $f \in L$ by Lemma 3.1. This proves the converse inclusion $Z^{\leftarrow}[Z[L]] \subseteq L$.

As a consequence of Theorem 3.6 we note that for an affine transformation $f$ with nonempty zero-set $Z(f)$ the left ideal $\langle f\rangle_{\ell}$ generated by $f$ and the annihilator Ann $(Z(f))$ of $Z(f)$ coincide. Furthermore, we get the following

COROLLARY 3.7. The not invariant left invariant ideals $L$ of $\mathrm{Aff}(V)$ are precisely the sets

$$
Z^{\leftarrow}[\mathcal{F}]=\bigcup\{\operatorname{Ann}(A) \mid A \in \mathcal{F}\}
$$

where $\mathcal{F}$ is a filter of affine subspaces of $V$.

\section{THE FINITELY GENERATED LEFT IDEALS}

Now we are in a position to determine the finitely generated left ideals of $\mathrm{Aff}(V)$.

THEOREM 4.1. Let $V$ be a vector space.

1. The finitely generated left invariant left ideals of $\mathrm{Aff}(V)$ are precisely the sets $\operatorname{Ann}(U)+C$, where $U$ is a linear subspace of $V$.

2. The finitely generated left ideals of $\operatorname{Aff}(V)$, which are not left invariant, are precisely the annihilators $\operatorname{Ann}(A)$, where $A$ is an affine subspace of $V$.

Proof: The first assertion of the theorem follows by Theorem $A$ in [1, p.173], Theorem 1 in [1, p.177], the second isomorphism theorem for near-rings and Lemma 2.2. To show 2 , suppose first that $L=\left(f_{1}, \ldots, f_{n}\right\rangle_{\ell}$ is a finitely generated left ideal of $\operatorname{Aff}(V)$ which is not left invariant. By Theorem 3.6 the family $Z[L]$ is an $\mathcal{A}$-filter on $V$. Hence there exists $f \in L$ with $Z(f)=Z\left(f_{1}\right) \cap \cdots \cap Z\left(f_{n}\right)$. Moreover, $Z(f)$ is not empty. By the remarks following Theorem 3.6 the left ideal $\langle f\rangle_{\ell}$ generated by $f$ agrees with the annihilator $\operatorname{Ann}(Z(f))$. Therefore $\operatorname{Ann}(Z(f)) \subseteq L$. Since $\operatorname{Ann}(Z(f))$ is a left ideal of $\operatorname{Aff}(V)$ containing $f_{1}, \ldots, f_{n}$, it follows that $\operatorname{Ann}(Z(f))=L$. 
Conversely, if $A$ is an affine subspace of $V$, by Lemma 2.4 there exists $f \in \operatorname{Aff}(V)$ with $A=Z(f)$. The remarks following Theorem 3.6 imply $\operatorname{Ann}(A)=\langle f\rangle_{\ell}$, hence $\operatorname{Ann}(A)$ is a finitely generated and obviously not left invariant left ideal of $\operatorname{Aff}(V)$.

For the proof of the next theorem it will be convenient to have

LEMMA 4.2. Let $V$ be a vector space. Then the following statements are equivalent:

1. $\operatorname{dim} V<\infty$.

2. Every $\mathcal{A}$-filter on $V$ is generated by an affine subspace of $V$.

Proof: Let $\operatorname{dim} V<\infty$ and let $\mathcal{F}$ be an $\mathcal{A}$-filter on $V$. Let $A \in \mathcal{F}$ such that $\operatorname{dim} A \leqslant \operatorname{dim} A^{\prime}$ for all $A^{\prime} \in \mathcal{F}$. If $A^{\prime} \in \mathcal{F}$, then $A \cap A^{\prime} \in \mathcal{F}$ and so $\operatorname{dim} A \leqslant$ $\operatorname{dim}\left(A \cap A^{\prime}\right)$. This implies $A \subseteq A^{\prime}$. Hence $\mathcal{F}$ is contained in the $\mathcal{A}$-filter $\mathcal{F}_{A}$ generated by $A$. Since $A \in \mathcal{F}$, it follows that $\mathcal{F}=\mathcal{F}_{A}$.

To show the converse, suppose that $\operatorname{dim} V=\infty$. Then the family of all finite dimensional linear subspaces of $V$ is an $\mathcal{A}$-filter on $V$ which is not generated by an affine subspace of $V$.

THEOREM 4.3. Let $V$ be a vector space. Then the following statements are equivalent:

1. $\operatorname{dim} V<\infty$.

2. All left ideals of $\mathrm{Aff}(V)$ are finitely generated.

Proof: Let $V$ be a finite dimensional vector space and let $L$ be a left ideal of $\operatorname{Aff}(V)$. If $L$ is not left invariant, then according to Corollary 3.7 and Lemma 4.2 there exists an affine subspace $A$ of $V$ with $L=\bigcup\left\{\operatorname{Ann}\left(A^{\prime}\right) \mid A^{\prime} \in \mathcal{F}_{A}\right\}=\operatorname{Ann}(A)$. Therefore Theorem 4.1 implies that $L$ is finitely generated.

If $L$ is a left invariant left ideal of $\operatorname{Aff}(V)$, then $L$ can be decomposed as $L=$ $L_{0}+C$, where $L_{0}$ is a left ideal of $\operatorname{Hom}(V, V)$. In particular, $L_{0}$ is a left ideal of $\operatorname{Aff}(V)$ which is not left invariant. Hence $L_{0}$ is finitely generated. Furthermore, Lemma 2.3 implies that $C$ is a finitely generated left ideal of $\operatorname{Aff}(V)$. Therefore $L$ is finitely generated.

If conversely all left ideals of $\mathrm{Aff}(V)$ are finitely generated, then $\operatorname{dim} V<\infty$ by Theorem 4.1, Lemma 4.2 and Corollary 3.7.

In particular, Theorem 4.1 and Theorem 4.3 show that for a finite dimensional vector space $V$ there is a Galois correspondence between the left invariant left ideals of $\mathrm{Aff}(V)$ and the linear subspaces of $V$ and a similar correspondence between the not left invariant left ideals of $\mathrm{Aff}(V)$ and the affine subspaces of $V$. 


\section{REFERENCES}

[1] R. Brer, Linear Algebra and Projective Geometry (Academic Press Inc., New York, 1952).

[2] D.W. Blackett, 'The near-ring of affine transformations', Proc. A mer. Math. Soc. 7 (1956), 517-519.

[3] J.D.P. Meldrum, Near-rings and their links with groups: Research Notes in Mathematics 134 (Pitman, Boston, London, Melbourne, 1985).

[4] G. Pilz, Near-rings: Mathematics Studies 23 (North Holland, New York, 1983).

[5] K.G. Wolfson, 'Two-sided ideals of the affine near-ring', Amer. Math. Monthly 65 (1958), 29-30.

Mathematisches Institut

Universität Erlangen-Nürnberg

Bismarckstr. $1 \frac{1}{2}$

D-8520 Erlangen,

Federal Republic of Germany 